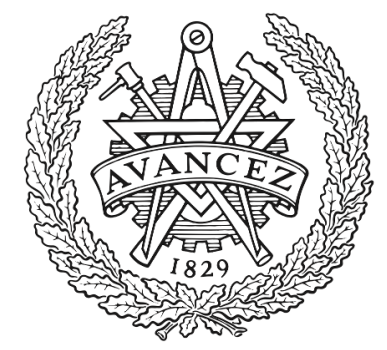

\title{
CHALMERS
}

UNIVERSITY OF TECHNOLOGY

\section{Structural Comparison between Sucrose and Trehalose in Aqueous Solution}

Downloaded from: https://research.chalmers.se, 2023-04-26 09:24 UTC

Citation for the original published paper (version of record):

Olsson, C., Swenson, J. (2020). Structural Comparison between Sucrose and Trehalose in Aqueous

Solution. Journal of Physical Chemistry B, 124(15): 3074-3082.

http://dx.doi.org/10.1021/acs.jpcb.9b09701

N.B. When citing this work, cite the original published paper. 


\title{
Structural Comparison between Sucrose and Trehalose in Aqueous Solution
}

\author{
Christoffer Olsson* and Jan Swenson
}

Cite This: J. Phys. Chem. B 2020, 124, 3074-3082

Read Online

ABSTRACT: The two sugar molecules sucrose and trehalose are both considered as stabilizing molecules for the purpose of preserving biological materials during, for example, lyophilization or cryo-preservation. Although these molecules share a similar molecular structure, there are several important differences in their properties when they interact with water, such as differences in solubility, viscosity, and glass transition temperature. In general, trehalose has been shown to be more efficient than other sugar molecules in preserving different biological molecules against stress, and thus by investigating how these two disaccharides differ in their water interaction, it is possible to further understand what makes

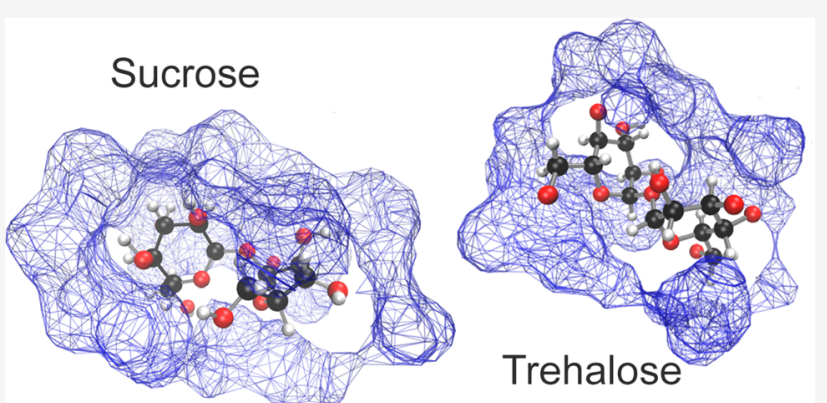
trehalose special in its stabilizing properties. For this purpose, the structure of aqueous solutions of these disaccharides was studied by using neutron and X-ray diffraction in combination with empirical potential structure refinement (EPSR) modeling. The results show that there are surprisingly few differences in the overall structure of the solutions, although there are indications for that trehalose perturbs the water structure slightly more than sucrose.

\section{INTRODUCTION}

In a previous study we examined the structural properties of an aqueous trehalose solution for the purpose of investigating its role as a protein stabilizer. ${ }^{1}$ The main results showed that trehalose forms multiple (although weak) bonds with water, avoiding direct trehalose-trehalose interactions (clustering), and had a destructuring effect on the water. However, the results obtained in that study did not include any comparisons with other sugar molecules. Thus, the question is whether our findings are unique for trehalose and therefore may explain its special stabilizing properties or if similar results would be obtained for other sugar molecules, such as sucrose.

Sucrose and trehalose are both disaccharide molecules with the same chemical formula $\left(\mathrm{C}_{12} \mathrm{H}_{22} \mathrm{O}_{11}\right)$ but with different geometrical structures (see Figure 1). The sucrose molecule consists of one glucose ring, and one fructose ring, connected by a glycosidic linkage, whereas the trehalose molecule consists of two glycosidic-linked glucose rings.<smiles>OCC1OC(OC2(CO)OC(CO)C(O)C2O)C(O)C(O)C1O</smiles><smiles>OCC1OC(OC2OC(CO)C(O)C(O)C2O)C(O)C(O)C1O</smiles>

Figure 1. Simple molecular sketches of sucrose (left) and trehalose (right). The carbon-bound hydrogens (labeled "M") have been omitted from the drawings for clarity.
Both sucrose and trehalose are often used as a stabilizing agent for different storing purposes, and it is commonly known that disaccharides in general have stabilizing effects both for the purpose of stabilization during cryo-storage and for the purpose of anhydrobiotic preservation (e.g., freeze-drying). ${ }^{2}$ When comparing the disaccharides trehalose and sucrose, however, it is found that trehalose is superior for many different biological preservation purposes, ${ }^{3,4}$ such as stabilization against thermal stress ${ }^{5,6}$ or during lyophilization. ${ }^{7-9}$ It is therefore of interest to determine what aspects of these rather similar molecules are important for biological stabilization. There have been plenty of studies comparing the two types of molecules for different purposes. ${ }^{5,10-16}$ On a macroscopic level there are some obvious differences: For example, sucrose has an exceptionally high solubility in water of $2.14 \mathrm{~g}$ of sucrose per $1 \mathrm{~g}$ of water (which implies that only 9 water molecules per sucrose molecule are required to dissolve the sugar), whereas trehalose can only be dissolved up to $1.1 \mathrm{~g}$ of trehalose per $1 \mathrm{~g}$ of water. ${ }^{17}$ Possibly one of the most important differences of these two sugar molecules, for preservation purposes, is the larger glass transition temperature $\left(T_{\mathrm{g}}\right)$ of trehalose compared

Received: October 15, 2019

Revised: $\quad$ March 26, 2020

Published: March 30, 2020 
to sucrose. ${ }^{5,18,19}$ This difference relates to the higher ability of trehalose to form a homogeneous amorphous network ${ }^{14,20}$ which, in turn, leads to a slowing down of the water dynamics $^{21}$ and perhaps, more importantly, to its larger capability to incorporate water molecules into its network, thus preventing water from crystallizing. ${ }^{5}$ Furthermore, $T_{\mathrm{g}}$ of a trehalose-water matrix has been shown to decrease less with an addition of small amounts of water compared to, for example, sucrose. $^{22-24}$ This implies that trehalose provides a more stable environment for the biological molecules and is better at maintaining a more rigid vitrified surrounding. ${ }^{25}$

The questions are, however, what the structural differences are that produce these differences in macroscopic properties. Several groups have examined the number of bonds formed between the different disaccharides, and some of them have concluded that trehalose forms more and stronger hydrogen bonds with water than, for example, sucrose. This has been shown by the use of, for example, ultrasonic velocity measurements, ${ }^{26}$ quasi-elastic neutron scattering (QENS) measurements, ${ }^{27}$ and molecular dynamics (MD) simulations. ${ }^{14}$ Lerbret et al. ${ }^{14}$ have furthermore pointed out that trehalose, compared to sucrose and maltose, retains a less folded structure when dehydrated and tends to avoid cluster formation in an aqueous solution (which was also found in ref 1 ), thus exposing more of its moieties to the surrounding water molecules. However, other groups point out that the total hydration numbers of sucrose and trehalose are actually quite similar. $^{12,13}$

Another important property of disaccharides is that they tend to have a destructuring effect on the natural tetrahedral structure of water. ${ }^{10,12}$ This destructuring effect has been found to be particularly strong for trehalose, ${ }^{10,14,21,28-32}$ and this observation is often used to explain parts of the extraordinary stabilizing properties of trehalose. However, Soper and co-workers have disputed this argument by showing very small destructuring effects on the water by the use of neutron and X-ray diffraction combined with empirical potential structure refinement (EPSR) modeling. ${ }^{33,34}$

In this paper we extend our previous study ${ }^{1}$ on an aqueous trehalose solution by also studying sucrose at the same solution concentration ( $33 \mathrm{wt} \%)$ also with the use of both neutron and $\mathrm{X}$-ray diffraction combined with EPSR modeling. From this we can directly compare the effect the two sugars have on their aqueous environment and also how the sugars themselves interact and structure in water. The purpose of this is to elucidate the aspects that make trehalose better at, for example, stabilizing proteins. However, the present results indicate very small structural differences between the sucrose and the trehalose solutions. A new EPSR model for trehalose has been produced, which showed a slightly smaller effect on the water structure than what was found in our previous study, ${ }^{1}$ thus more in line with the similar studies made by Pagnotta et al. ${ }^{33}$ and Soper et al. ${ }^{34}$ However, by comparison with sucrose, it was found that the presence of trehalose appears to have a stronger effect on the structure of bulk water than sucrose. A similar abundance of water-disaccharide bonds was found in this study as in our previous study on only trehalose. ${ }^{1}$

\section{EXPERIMENTAL SECTION}

Sample Preparation. All sucrose samples were prepared in the same way as trehalose in ref 1 . Six samples with different deuterations were made with the same molar concentration of 38 water molecules per sucrose (corresponding to a sugar content of $33 \mathrm{wt} \%$ for the fully hydrogenous sample). The sucrose was dissolved in either $\mathrm{H}_{2} \mathrm{O}, \mathrm{D}_{2} \mathrm{O}$, or a $1: 1$ molar concentration mixture of the two (HDO). Two different isotope conformations of sucrose were used: either regular hydrogenated sucrose (purchased in crystalline form from Sigma-Aldrich, with $>99.5 \%$ purity), which will be denoted as $\mathrm{H}$-Sucrose (or H-Suc), or deuterated sucrose, in which the 14 carbon-bound hydrogens had been exchanged for deuterium (purchased in crystal form from Omicrometer Biochemicals Inc.) which will be denoted as D-Sucrose (or D-Suc). The hydrogens at the hydroxyl groups are exchangeable in water, and therefore these hydrogens were replaced with deuterium by repeatedly dissolving and drying (under vacuum at $70{ }^{\circ} \mathrm{C}$ ) the sucrose in either $\mathrm{D}_{2} \mathrm{O}$ or $\mathrm{HDO}$ (depending on what solvent they were to be finally dissolved in). This procedure was used to ensure that the exchangeable sucrose hydrogens always had the same isotope composition as the solvent. The six different isotope compositions were either D-Sucrose in $\mathrm{H}_{2} \mathrm{O}, \mathrm{D}_{2} \mathrm{O}$, or HDO (D-Suc $\mathrm{D}_{2} \mathrm{O}, \mathrm{D}$-Suc $\mathrm{H}_{2} \mathrm{O}$, and D-Suc $\mathrm{HDO})$, or $\mathrm{H}$-Sucrose in $\mathrm{H}_{2} \mathrm{O}, \mathrm{D}_{2} \mathrm{O}$, or $\mathrm{HDO}\left(\mathrm{H}-\mathrm{Suc} \mathrm{D}_{2} \mathrm{O}, \mathrm{H}\right.$ Suc $\mathrm{H}_{2} \mathrm{O}$, and $\mathrm{H}$-Suc HDO). The neutron diffraction data of trehalose $\left(\alpha, \alpha\right.$-trehalose) was taken from our previous study; ${ }^{1}$ however, we now added X-ray diffraction data of fully protonated trehalose in $\mathrm{H}_{2} \mathrm{O}\left(\mathrm{H}\right.$-Tre in $\left.\mathrm{H}_{2} \mathrm{O}\right)$, prepared in the same way as described in ref 1 .

Neutron Diffraction Experiments. The neutron diffraction measurements and data corrections were performed by using the same method as described in ref 1 . The samples were placed, using a syringe, inside $1 \mathrm{~mm}$ thick $\mathrm{Ti}_{0.676} \mathrm{Zr}_{0.324}$ containers, which were sealed with a PTFE O-ring. These containers were mounted on a sample changer with a water bath temperature control set to $298 \mathrm{~K}$. The measurements were performed on the NIMROD diffractometer ${ }^{35}$ at the ISIS neutron spallation source (Rutherford Appleton Laboratory, UK).

Once the data were collected, it was corrected (e.g., background subtraction and inelastic scattering corrections) by using the GUDRUN suite (2015 version). ${ }^{36}$ From these corrections the interference differential cross sections $(I(Q))$ for the sample were obtained:

$$
I(Q)=\sum_{\alpha, \beta}\left(2-\delta_{\alpha, \beta}\right) c_{\alpha} c_{\beta} b_{\alpha} b_{\beta}^{*}\left(S_{\alpha, \beta}(Q)-1\right)
$$

where $Q$ is the neutron momentum transfer, $c_{\alpha}$ is the number density of atom type $\alpha, b_{\alpha}$ is the corresponding scattering length of that atom, and $S_{\alpha, \beta}(Q)$ is the partial structure factor of atom types $\alpha$ and $\beta$, i.e., describing the correlations between these two atoms.

X-ray Experiments. The samples taken for X-ray measurements were prepared in the same way as for the fully protonated neutron diffraction samples $\left(\mathrm{H}\right.$-Sugar and $\mathrm{H}_{2} \mathrm{O}$ ). Samples were placed in $2 \mathrm{~mm}$ wide silica glass capillaries and placed in an Empyrean X-ray diffractometer (using an $\mathrm{Ag}$ anode) with an X-ray beam with a wavelength of 0.5609 A. The data were corrected for background scattering, multiple scattering, and attenuation and converted to an interference differential cross section scale with the use of GUDRUN-X, which is extensively explained in ref 37.

EPSR Modeling. The corrected data were fitted by using EPSR modeling. ${ }^{38}$ In this method, a molecular simulation box is created with the identical chemical composition as the measured sample. The user also assigns an appropriate reference potential for the different atom types (typically 
based on known force-field parameters), and then the software calculates the theoretical $I(Q)$ for the different isotope compositions and compares those to the experimental data. An empirical potential, based on the difference between supplied and simulated $I(Q)$, is then added to the reference potential, which subsequently reconfigures the molecular model.

Theoretical details regarding the empirical potential structure refinement (EPSR) method are well described in, for examle, ref 38 . The simulation was set up in a similar way as in ref 1 , although with some important differences. The simulation box was doubled in size, with 4000 water molecules and 104 sucrose molecules. For comparison, the previously produced trehalose simulation from ref 1 was rerun with the same increased box size and with the addition of X-ray data. The reference potential for sucrose was set identical to that of trehalose, which was based on the parameters used in ref 33 (which in turn was based on the OPLS-AA force field ${ }^{39}$ for trehalose and the SPC/E model for the water molecules ${ }^{40}$ ). The reference potential for the new trehalose EPSR model was set identical to our previous study. ${ }^{1}$ Details regarding the force field parameters for the reference potential can be seen in Table S1 of the Supporting Information. The atomic labeling of sucrose was mainly set analogous to that of trehalose in ref 1 (see Figure 1) and ref 33; however, an additional oxygen type (O4) was defined for the hydroxymethyl groups on the fructose ring of the sucrose molecule. This was introduced to be able to distinguish between the two sugar rings (glucose and fructose) for at least one atom type. The number density of the model for the sucrose sample was set to 0.106439, as determined by a density measurement of the fully hydrogenated sucrose sample, and 0.10677 for the trehalose model, as previously used in ref 1 .

After the simulation box was equilibrated by using the reference potential, the diffraction data were introduced to refine the empirical potential. The influence of the empirical potential was increased until the fit with the diffraction data was no longer improved. When the fit reached this point, statistics about the systems was gathered by collecting $\sim 1000$ molecular configurations. Average molecular properties presented in the results originate from these configurations.

EPSR Analysis. From the obtained molecular model, different coordination numbers from atom $\alpha$ to atom $\beta$ (see Table S2) were calculated via the following equation:

$$
n_{\alpha}^{\beta}=4 \pi c_{\beta} \int_{r_{1}}^{r_{2}} g_{\alpha, \beta}(r) r^{2} \mathrm{~d} r
$$

where $g_{\alpha, \beta}(r)$ is the partial pair correlation function of atoms $\alpha$ and $\beta$ and $c_{\beta}$ is the atomic number density of atom $\beta$. The integration is done between the center of the atom $\alpha\left(r_{1}=0\right)$ and a certain distance $\left(r_{2}\right.$, where $r_{2}$ depends on which coordination number is of interest).

Hydrogen bonds were also calculated by three different hydrogen-bond criteria. In criteria 1 a hydrogen bond is defined as soon as the distance of a hydrogen and an oxygen is $<2.5 \AA$ (as used in e.g. refs 41-43) and thus contains no constraint regarding the bond angles of relevance. Criteria 2 and 3 define a hydrogen bond when the distance between two oxygen atoms is $<3.4 \AA$ and the angle between donorhydrogen-acceptor is above $120^{\circ}$ and $160^{\circ}$, respectively. Criteria 2 was used in, for example, refs 12, 14, and 40, and the more strict definition of a hydrogen bond, criteria 3, was also used in ref 14 . The hydrogen bond analysis for criteria 2 and 3 was done by creating a "pseudo-trajectory" from the EPSR model of $\sim 500$ configurations and analyzing this trajectory by using the molecular dynamics trajectory analysis software VMD. ${ }^{45}$

SANS Fits. To determine the size distribution of the two different disaccharides, a separate fit was made to the smallangle scattering part (the Q-range $0.05-1 \AA^{-1}$ ) of the diffraction data. Specifically, the fits were made on either DSuc in $\mathrm{H}_{2} \mathrm{O}$ or D-Suc in HDO (and D-Tre in $\mathrm{H}_{2} \mathrm{O}$ and D-Tre in $\mathrm{HDO}$ ) because these data sets have the strongest form factor contribution to the signal. The fits were made with the help of the SasView software (v. 4.1.2). ${ }^{46}$ Simultaneous fits of the two different isotope compositions were performed by using a spherical hard-sphere model with a Gaussian distribution of the radius. The scattering length densities for the solute and the solvent for the two different isotope compositions were fixated, and similarly, the volume fraction of the disaccharide molecules was fixed and approximated to 0.2467 , based on the macroscopic densities of the two components in the solution. The $Q$-range was selected so that it captures the steep descent of the $I(Q)$ signals, which was assumed to originate mainly from the form factor of the disaccharides.

\section{RESULTS}

Q-Space Analysis. $I(Q)$ of both sucrose and trehalose solutions of all isotope compositions are shown in Figure 2. It

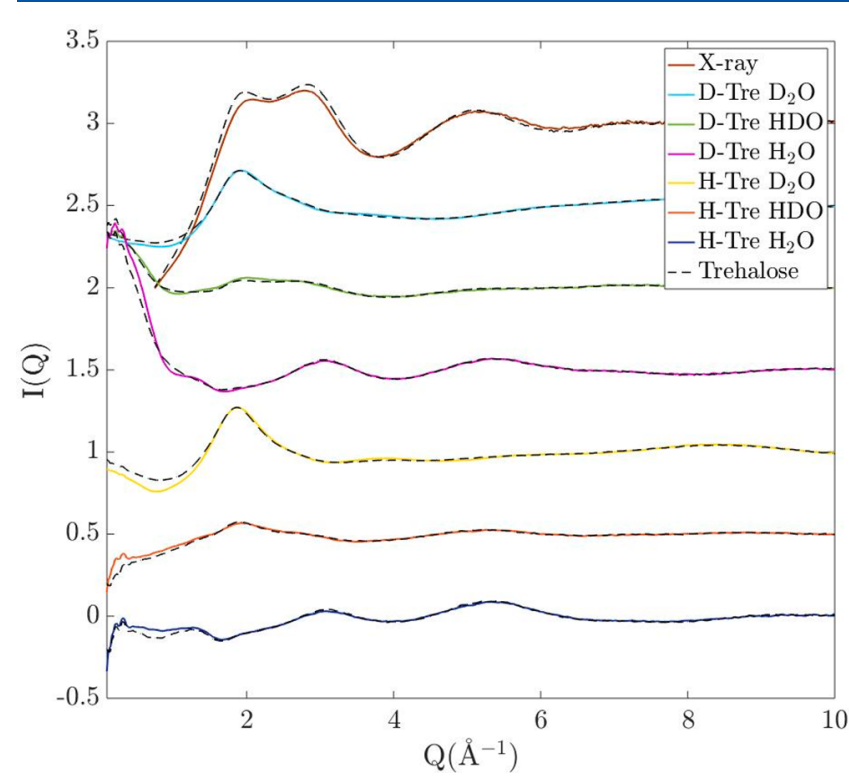

Figure 2. Differential cross sections, $I(Q)$, of both sucrose and trehalose in a $1.1 \mathrm{M}$ aqueous solution. Full lines show $I(Q)$ of sucrose solutions of different isotope compositions, and black dashed lines show $I(Q)$ for the same isotope compositions of the trehalose solutions.

should be noted that some of the differences, namely, the overall level of these curves, may differ slightly due to inaccuracies in the data correction steps, such as in the inelasticity correction. This particularly becomes more problematic with higher fractions of hydrogens and at lower $Q$ 's. The data sets coming from the two different disaccharides are, however, very similar, although some features are noticeably different. In Figure 3, these differences are amplified and are plotted in comparison to bulk water $\left(\mathrm{H}_{2} \mathrm{O}, \mathrm{HDO}\right.$, or 

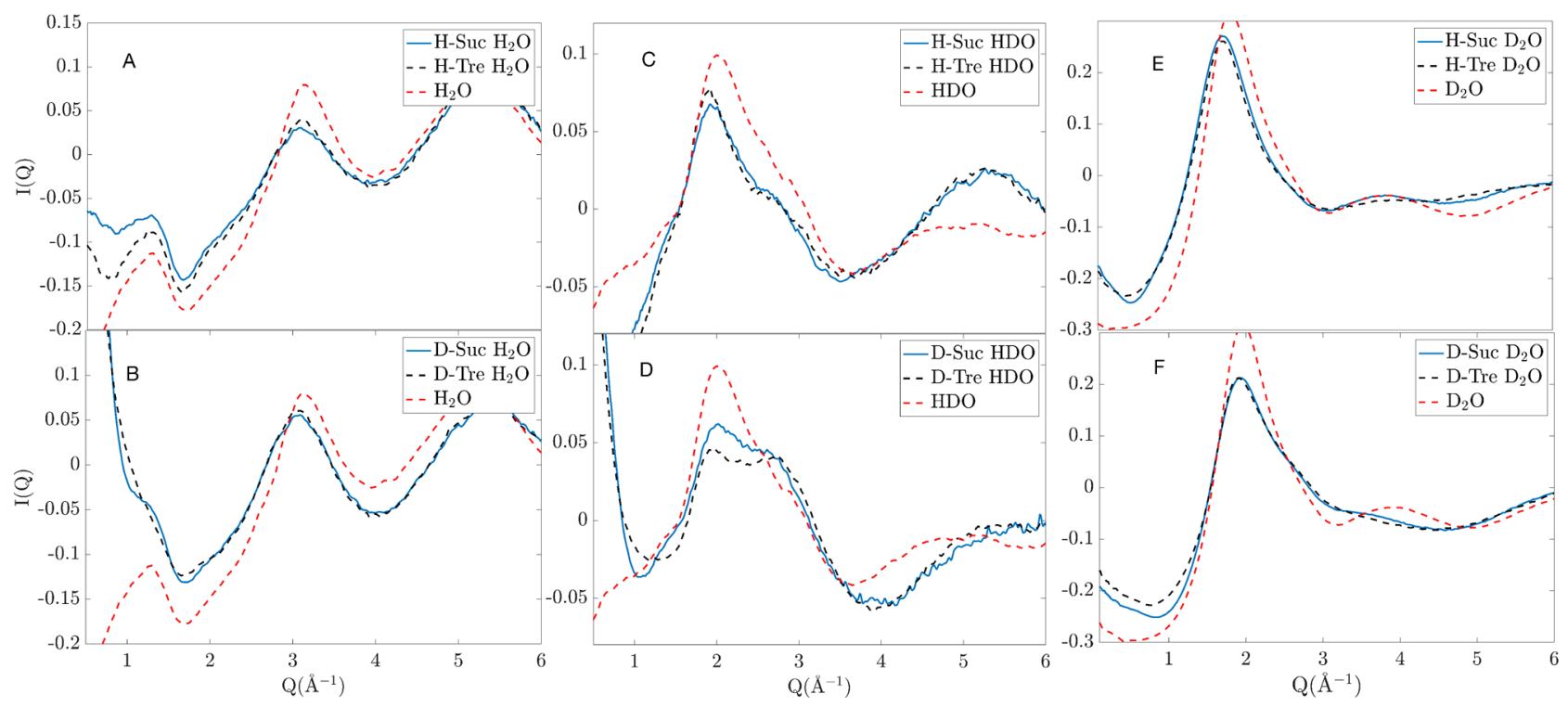

Figure 3. Zoomed-in differential scattering cross sections for different isotope compositions compared with different isotope compositions of bulk water at the same temperature. Blue lines represent sucrose, black dashed lines represent trehalose, and red dashed-dotted lines represent bulk water. Corresponding differential scattering cross section from X-ray measurement can be found in Figure S1.

$\left.\mathrm{D}_{2} \mathrm{O}\right)$. These bulk-water data were obtained from ref 47 and were converted to the same scale as the sugar data sets. The most obvious feature differences between the different data sets are listed in the following points:

1. In the $\mathrm{H}-\mathrm{Suc} \mathrm{H}_{2} \mathrm{O}$ data (Figure $3 \mathrm{~A}$ ), there are few clear differences. Trehalose exhibits a slightly larger peak than sucrose around $3 \AA^{-1}$.

2. In the $\mathrm{D}$-Suc $\mathrm{H}_{2} \mathrm{O}$ data (Figure $3 \mathrm{~B}$ ), sucrose shows a steeper descent in the region $0.2-1 \AA^{-1}$ and exhibits a small peak around $1.25 \AA^{-1}$, which is not present in the trehalose data.

3. In the H-Suc HDO data (Figure 3C), there are few clear differences. Trehalose exhibits a slightly larger peak than sucrose around $2 \AA^{-1}$.

4. In the D-Suc HDO data (Figure 3D), sucrose exhibits a small shoulder between 1.2 and $1.6 \AA^{-1}$, which is not present in the trehalose data. Furthermore, the sucrose data show a stronger peak at $2 \AA^{-1}$, but trehalose exhibits a more pronounced peak at $2.7 \AA^{-1}$. In this latter case, sucrose has a similar curve as the bulk water.

5. In the $\mathrm{H}$-Suc $\mathrm{D}_{2} \mathrm{O}$ data (Figure $3 \mathrm{E}$ ), sucrose features a more pronounced peak at $3.9 \AA^{-1}$ than trehalose. An even more pronounced peak, although quite similar to sucrose, is observed for bulk water at a similar $Q$-value.

6. In the D-Suc $\mathrm{D}_{2} \mathrm{O}$ data (Figure $3 \mathrm{~F}$ ), sucrose exhibits a weak peak at $3.65 \AA^{-1}$, which is not present in the trehalose data. A similar peak is present in the bulk water data.

The steeper descent (point 3) is most likely due to differences in the form factors between the different samples, which are related to the size of the individual sugar molecules (see the Results and Discussion sections). In general, however, it should be pointed out that the differences between the $I(Q)$ 's of the sucrose and trehalose solutions are quite small, but where they differ, it appears that the features of the sucrose solution are closer to those of bulk water compared to the corresponding features of the trehalose solution. These results indicate that the trehalose has a stronger effect on perturbing the structure of the water from its bulk-like properties.

Small-Angle Data Fitting. To determine the size of the individual sugar molecules, we fitted the small-angle data (in the range $0.05-1 \AA^{-1}$ ) of D-Suc $\mathrm{H}_{2} \mathrm{O}$ and D-Suc HDO (same isotope compositions for trehalose). The resulting fits can be seen in Figure 4 and resulted in a radius of $4.0 \AA$ for sucrose and $4.2 \AA$ for trehalose.

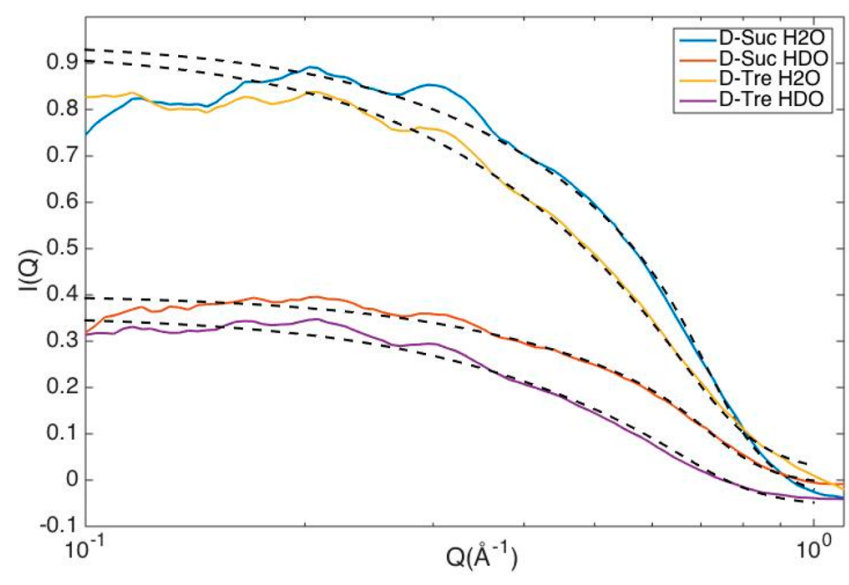

Figure 4. Small-angle data fitted for samples containing deuterated disaccharides in either $\mathrm{H}_{2} \mathrm{O}$ or HDO. Dotted lines show the fits to the data.

Furthermore, no significant clustering of either of the disaccharide molecules was found, as also obtained in our previous study on only trehalose. ${ }^{1}$ This can be seen directly from Figure 2 by the lack of any significant small-angle scattering except that from the individual disaccharide molecules (as highlighted in Figure 4). We also calculated cluster distributions from the EPSR models as seen in Figure 5. For this cluster distribution calculation two disaccharides were classified as clustered if any of the disaccharide oxygens reside within $2.5 \AA$ of any other disaccharide hydrogen. Noticeably, 


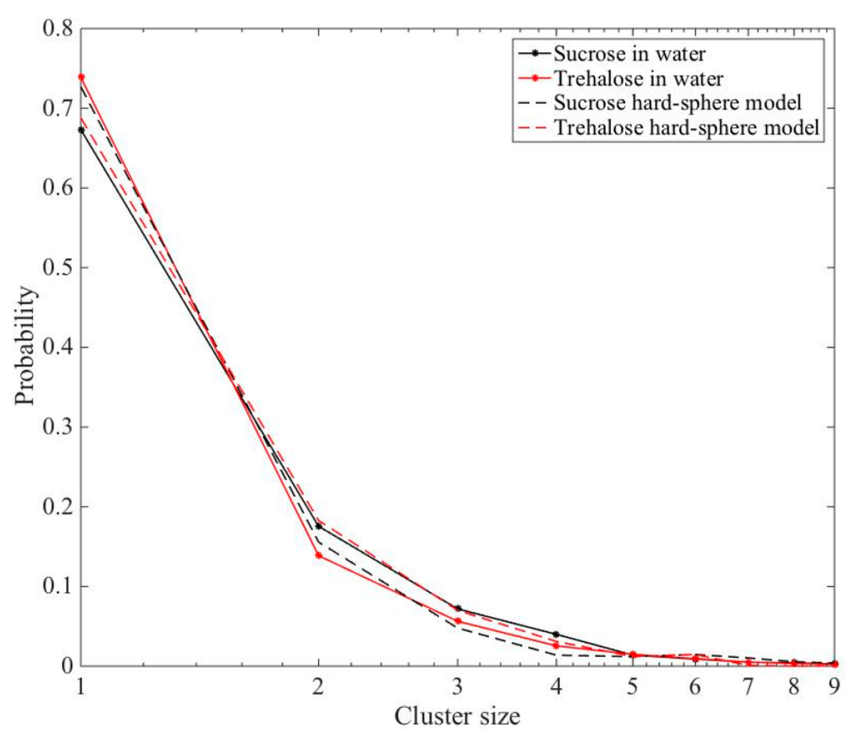

Figure 5. Cluster size distribution of sucrose and trehalose in water. Cluster criteria is defined here as when any of the hydroxyl oxygens of the disaccharide is within $2.5 \AA$ of an exchangeable hydrogen of any other disaccharide. Dashed lines represent a hard-sphere model of both disaccharides in water.

both disaccharides have a similar cluster distribution, where most $(\sim 80 \%)$ of the molecules are only bonding to water molecules. A hard-sphere model was produced in EPSR, where all potential parameters were set to zero (except from a minimum atom-atom distance constraint of $0.8 \AA$ to avoid atomic overlaps), for comparison. The hard-sphere model represents a system where all disaccharides are distributed randomly in the solution and can be seen to exhibit more "clustering" a than the actual EPSR models. This finding indicates that there is a preference against the formation of clusters of disaccharide molecules in both solutions.

EPSR Fits. The resulting EPSR fits to the total differential scattering cross sections are shown in Figure S2 for sucrose and in Figure S3 for trehalose. We furthermore also fitted the model to the first order difference of $I_{\mathrm{DDD}}(Q)-I_{\mathrm{HDD}}(Q)$, which is shown in Figure 6 (Fourier transformed to a real space representation). This differential scattering cross section only contains correlations to the nonexchangeable hydrogen atoms ( $\mathrm{M}$ atoms), as discussed in greater detail below. Because of the dominating signal of water-water interactions in the total differential cross sections, this added differential scattering cross section forces the EPSR model to also take the relatively small signal of the $M$ atoms into account.

Although the fits capture most features of the experimentally obtained diffraction data and are in relatively good agreement (compared to e.g. previous mentioned studies on trehalose $\left.\mathrm{e}^{1,33,34,41}\right)$, there are still several discrepancies with the experimental data. These discrepancies are definitely larger than the experimentally measured differences between sucrose and trehalose. In fact, the total root-mean-square deviation between the EPSR fits and sucrose or trehalose is 0.23 and 0.27 respectively, whereas the total root-mean-square deviation between sucrose and trehalose is 0.13 . For this reason, we stress that no clear conclusions can be drawn from only EPSR modeling regarding structural differences of the two solutions. However, despite this accuracy problem, it is clear from the analysis presented below that EPSR can at least qualitatively reproduce the experimentally observed structural differences

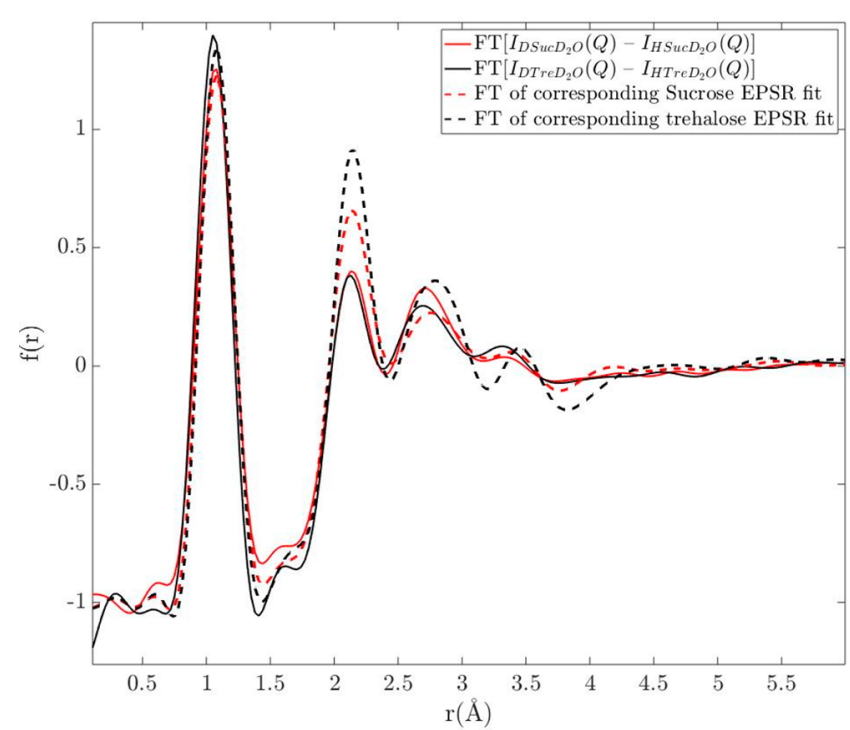

Figure 6. Correlation functions obtained from the Fourier transform of the difference between the differential scattering cross sections from $\mathrm{D}$-Sug in $\mathrm{D}_{2} \mathrm{O}$ and $\mathrm{H}$-Sug in $\mathrm{D}_{2} \mathrm{O}$ (red solid line for sucrose and black solid line for trehalose). Red and black dashed lines represent the Fourier transform of the corresponding EPSR fits for sucrose and trehalose, respectively. The first peak in $f(r)$ at about $1.1 \AA$ is entirely due to intramolecular $\mathrm{C}-\mathrm{M}$ correlations and consequently not related to the hydration of the disaccharides.

between the two solutions. The reason for this is most likely that the experimental data contain some experimental (or data correction) errors which makes it impossible to obtain full quantitative agreement with the data obtained for all the isotopically different samples (including X-ray diffraction data). Thus, the fits to the experimental data are not excellent, but nevertheless the produced models seem to be able to qualitatively reproduce most of the experimentally observed structural differences between the two solutions. Furthermore, both disaccharide solution models have been rerun several times, giving almost identical results. For example, the models consistently produce a larger distortion of the water structure in the case of trehalose (as seen in Figures S4 and S5), in agreement with direct experimental observations, as seen from the analysis of Figure 3 given above. However, because of the small structural differences between the two solutions in relationship to the uncertainties in the models, all the observed differences between the two sugar models cannot be regarded to be fully established and conclusive.

Nonetheless, quantitative results are valuable to obtain to allow us to compare our findings to previous works, such as those in refs $1,33,34$, and 41 . Within the limits of the error bars, our hydration numbers of trehalose (Table S2) are consistent with these previous studies, ${ }^{1,33,34,41}$ although our interpretation of the experimental results and models differs somewhat from those studies as further discussed below.

First-Order Difference Method. To compare more detailed information about the data with the model, a firstorder difference analysis method (as described in e.g. refs 48 and 49) was also used. In this method, the differential scattering cross sections of (presumably) identical chemical composition with differing isotope compositions are subtracted from each other. If the isotopes of a specific atom pair of the two different samples are identical, these are subsequently removed by the subtraction. In Figure 6, the differential 
scattering cross section of $\mathrm{H}$-Sug in $\mathrm{D}_{2} \mathrm{O}$ was subtracted from that of D-Sug in $\mathrm{D}_{2} \mathrm{O}$ and Fourier transformed to a real space representation; because all atoms except the $\mathrm{M}$ atoms of the sugars are identical, the only remaining pair correlations are those with the $\mathrm{M}$ atoms. The resulting correlation function thus hides water-water correlations and yields more information regarding water-sugar (54\% scattering contribution from $\mathrm{M}-\mathrm{Hw}$ and $23 \%$ from $\mathrm{M}-\mathrm{Ow}$ ) and sugar-sugar correlations (23\%). Although this method adds plenty of specific information regarding correlations with the $M$ atoms, which otherwise would be neglected by fitting the total scattering cross sections, it should be pointed out that this method is unfortunately quite sensitive to small errors in the data, which might come from the data corrections. Therefore, the small differences between the two experimentally obtained difference functions cannot be considered to be significant, but rather the small differences indicate that the sugar-water interactions are similar for the two solutions. The results from the EPSR modeling are in this case not fully consistent with the experimental findings, since the second and third peaks are stronger for trehalose due to stronger $\mathrm{M}$-water correlations.

A similar, second-order difference method was used to highlight the exchangeable hydrogen atoms in the sample, which is shown in Figure S5 (red and black dashed lines), compared to the $\mathrm{Hw}-\mathrm{Hw}$ pair correlations obtained from EPSR for both sugar solutions and with the $\mathrm{Hw}-\mathrm{Hw}$ pair correlation of bulk water (as obtained from refs 36 and 50). The pair correlations of the exchangeable hydrogen atoms were obtained by taking the Fourier transform of $\left(I_{\mathrm{DDD}}(Q)+\right.$ $\left.I_{\mathrm{DHH}}(Q)\right)-2\left(I_{\mathrm{DHD}}(Q)\right)$. By performing this slightly more complex subtraction, all correlations cancel out, except for the exchangeable hydrogen atoms ( $\mathrm{Hw}$ and $\mathrm{H}$ ). The majority (90\%) of this signal does, however, originate from the $\mathrm{Hw}-$ Hw atoms (since they are more abundant).

\section{DISCUSSION}

Throughout this section the reader should keep in mind that the $I(Q)$ 's indicate very minor differences between sucrose and trehalose (details on differences between the data sets can be seen in Figure 3), and, furthermore, the EPSR models are not able to reproduce the experimental data in all details. This means that there may be important features which the models fail to capture or, conversely, that features may be obtained that are not present in the data. That being said, if the models capture features that can be seen by a direct analysis of the experimental data (see Figure 3), it is a good indicator that the models have captured "true" features, and they can thus be used to quantify these features. Furthermore, these differences can also be corroborated by other studies, such as measurements of glass transitions, viscosity, and dynamical measurements, as discussed below.

Disaccharide-Water Interactions. A greater waterdestructuring effect of trehalose compared to sucrose has been previously shown in the literature..$^{10,14,21,28-32}$ This issue is important to explain the superior cryo-protective effect of trehalose. For example, a stronger destructuring effect should in principle lead to less crystallizable water, which could otherwise damage biological materials during cooling, and indeed it has been shown by, for example, calorimetric measurements that trehalose prevents more water from crystallization than sucrose. ${ }^{5}$ The results presented in Figure 3 clearly show that the intermediate structure (in the range 1$4 \AA^{-1}$ ) of these solutions is more similar to that of bulk water in the case of sucrose than in the case of trehalose, which is also supported from the EPSR model, as seen in Figure S4, for example. These results support the idea that trehalose perturbs the network structure of bulk water more efficiently than sucrose. The reason for this stronger destructuring effect has been suggested to be coupled to a couple of different, not necessarily opposing, ideas. One of these is that trehalose binds to more water molecules, as suggested by, for example, ref 26 . Another common hypothesis is that the way water binds to trehalose alters the three-dimensional structure of bulk water more than in the case of sucrose. ${ }^{16}$ Because the present experimental and EPSR results are not giving support for that there is any substantial difference in the number of water molecules trehalose and sucrose bind to, the latter explanation for the stronger destructuring effect of trehalose seems most plausible. Thus, the specific water-disaccharide interactions determine how the water molecules rearrange themselves around the disaccharide, which in turn determines how the water structure is perturbed. This observation has also been indicated in previous studies. ${ }^{12,13}$

Another important related aspect regarding how trehalose interacts with water is how it changes the dynamics of water. It has been shown, by multiple different studies, that trehalose exhibits a stronger dynamical coupling (i.e., longer residence times of a water-trehalose hydrogen bond and slower diffusion of water for trehalose than for other disaccharides). ${ }^{21,25,31,51}$ A stronger dynamic coupling indicates more and/or stronger hydrogen bonds between the two molecular species. From the present results it is, however, surprisingly difficult to determine any such differences since both disaccharides exhibit similar hydration numbers and similar hydrogen bonding distances to the atoms of the water molecules (see Figure 6, Figure S6, and Table S2). Thus, there is no obvious structural reason for the different dynamical properties ${ }^{21,25,31,51}$ of the two different sugar types.

A Note on the New EPSR Model of Trehalose and Water Structure. The new trehalose model produced in this work differs somewhat from our previous paper on trehalose in aqueous solution. ${ }^{1}$ As pointed out by Soper et al., ${ }^{34}$ X-ray diffraction data contain complementary information to neutron diffraction data, and particularly oxygen-oxygen correlations become more strongly weighted in X-ray diffraction data. Thus, the inclusion of X-ray diffraction data produces a more accurate model of the water structure in each sugar solution. Other differences for the present trehalose model are that we used a larger simulation box (4000:104 water:trehalose molecules instead of 2000:52) and that the influence of the empirical potential was constrained to a lower value in the current work. The main difference between this new model and our previous model is that the current model produced a slightly smaller effect on the structure of water than was previously obtained; thereby this result agrees more with what was obtained by Pagnotta et al. ${ }^{33}$ Soper et al. (coauthors of ref 33) performed a new study on aqueous trehalose which included the data provided from our own previous study ${ }^{1}$ and with the addition of X-ray data for the two trehalose concentrations provided in their previous paper. ${ }^{33}$ In their new study, ${ }^{34}$ they came to the same conclusion again that trehalose forms weak bonds with water and does not significantly perturb the water structure. Their obtained pair correlation functions are very similar to those presented for trehalose in this paper, and therefore we agree with their presented molecular model (see, for example, Figure $7 \mathrm{~b}$ of ref 
34 compared to Figure S5 for water-water correlations). We also partly agree with their conclusion that trehalose (and sucrose as seen here) form relatively weak bonds with water. However, their conclusions about the minor destructuring effect of trehalose differs somewhat from that presented here.

The partial pair correlation functions presented here for both trehalose and sucrose (Figure S6) are quite similar compared to those of previous studies on mannose ${ }^{52}$ or cellobiose $^{43}$ but indicate less bonding than compared to glucose. $^{52}$ It should however be pointed out that the concentrations in those studies differ from the one presented here, and therefore the results are not directly comparable. Nonetheless, compared to other sugar molecules neither trehalose nor sucrose exhibits extraordinary weak or strong water interactions. Furthermore, Soper et al. ${ }^{34}$ refer to a paper by Towey et al. ${ }^{42}$ in which the correlations between water and glycerol were found to be strong in comparison to the disaccharides presented here. However, Towey et al. claim in a later study ${ }^{53}$ that glycerol perturbs the typical tetrahedral structure of bulk water less than trehalose. They found that trehalose, which data were obtained from Pagnotta et al., ${ }^{33}$ shifts the second peak position of $g_{\text {OwOw }}$ more than glycerol (for about 20 water molecules per glycerol) and should thus be even more efficient in perturbing the tetrahedral water structure.

Soper et al. ${ }^{34}$ also bring up the issue that the first peak of $g_{\text {OwOw }}$ increases with the addition of trehalose. Indeed, such an effect can be seen also for sucrose, and it was brought up in ref 1 , too; however, it was pointed to that it is precisely this additional water in the first coordination shell of a water molecule which was proposed to give rise to the water destructuring effect. This is highlighted by Figure S4, showing that the addition of trehalose (and sucrose) promotes $\mathrm{O}-\mathrm{O}-$ $O$ triplet angles of $60^{\circ}$, corresponding to an equilateral triangular formation, ${ }^{54}$ rather than the tetrahedral structure at $109^{\circ}$. This is suggested to be due to the presence of an interstitial water molecule in the first coordination shell of the water. If some of the water molecules become relatively more packed, this should be associated with an increase in density, which indeed is indicated by the fact that the density of the aqueous trehalose solution is slightly higher than that of the sucrose solution at the same concentration and temperature ( 1.1296 and $1.1270 \mathrm{~g} / \mathrm{mL}$ for trehalose and sucrose, respectively).

Intra- and Interdisaccharide Interactions. Lerbret et al. ${ }^{14}$ have performed a set of simulations which suggest that trehalose has a more open structure in aqueous solutions than sucrose, that is, that sucrose forms more intramolecular hydrogen bonds. This has also previously been shown by Xray diffraction, ${ }^{55}$ where the authors showed that sucrose forms more intramolecular hydrogen bonds, exhibiting a closer more compact structure, as the concentration is increased above $\sim 22$ wt\% sucrose (below this concentration no intramolecular hydrogen bonds are formed). Here we study a sucrose solution of $33 \mathrm{wt} \%$ sucrose and thus expect, according to ref 55, that some, but few, intramolecular hydrogen bonds exist. The only clear indication of this was seen from the form factor analysis (see Figure 4), which shows that, on average, sucrose has a slightly smaller radius of gyration than trehalose, which indicates that the sucrose molecules are slightly more folded than the trehalose molecules.

The idea that sucrose forms more intramolecular bonds with increasing disaccharide concentration can also explain why sucrose has a significantly higher solubility than trehalose. In the low water limit, sucrose forms multiple intramolecular hydrogen bonds, ${ }^{55}$ whereas trehalose remains unfolded, and thus has more of its hydrogen-bonding sites available for interactions with other molecules. ${ }^{14}$ For this reason, sucrose only requires about 8.9 water molecules to cover its smaller interactive surface, whereas 17.3 water molecules are required to prevent trehalose molecules from directly interacting with each other. However, in this study, the disaccharide solutions were too diluted for these effects to be evident, and we can only conclude that if such a structural difference is present, it is more prominent at lower water concentrations.

\section{CONCLUSION}

It may be expected that the structural differences between aqueous sucrose and trehalose should be large due to their different macroscopic and dynamical properties. However, the diffraction data clearly exhibited only small discrepancies in the overall structures of the two solutions (see Figure 3). On the basis of the isotope subtraction method to highlight interactions with the carbon-bound hydrogen atoms ( $M$ atoms), as shown in Figure 6, it could be seen that the $M$ atoms of the two sugars bind to similar numbers of water molecules in the two solutions. Although the structural EPSR models, based on the differential scattering cross-section data, suggest that sucrose binds slightly more water molecules in total, the difference is so small that it cannot be established due to the uncertainties in the EPSR produced structural models. Furthermore, directly from the diffraction data, as seen in Figure 4, it was evident that there are no clear differences in intermolecular clustering of the sugar molecules, since neither sucrose nor trehalose shows a preference of forming clusters. The differences that were observed in the experimentally obtained scattering data do, however, provide key insights into the subtle differences these molecules exhibit. First of all, it was shown that the structure factor of bulk water is more similar to that of sucrose than to trehalose, thus indicating that trehalose has a stronger perturbing effect on the structure of water. Second, the overall radius of gyration was slightly larger for trehalose than for sucrose, and, finally, the water molecules seem to interact slightly differently with the different atomic sites of the two disaccharides. All these observations are in line with what has previously been shown by others ${ }^{10,14,21,28-31,55}$ and can partly explain why the solubility of sucrose is higher than for trehalose and, more importantly, provides possible explanations for why trehalose is superior to sucrose as a biological stabilizer.

\section{ASSOCIATED CONTENT}

\section{Supporting Information}

The Supporting Information is available free of charge at https://pubs.acs.org/doi/10.1021/acs.jpcb.9b09701.

Additional differential cross sections from X-ray scattering compared to bulk water; EPSR force field parameters; EPSR fits to experimental data; EPSR analysis of water-sugar and water-water interactions (PDF)

\section{AUTHOR INFORMATION}

\section{Corresponding Author}

Christoffer Olsson - Department of Physics, Chalmers University of Technology, SE-41296 Göteborg, Sweden; 
다이.org/0000-0002-8440-9393;

Email: christoffer.olsson@chalmers.se

\section{Author \\ Jan Swenson - Department of Physics, Chalmers University of

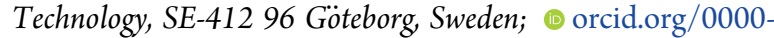 0001-5640-4766}

Complete contact information is available at:

https://pubs.acs.org/10.1021/acs.jpcb.9b09701

\section{Funding}

This work was financially supported by the Swedish Research Council.

\section{Notes}

The authors declare no competing financial interest.

\section{ACKNOWLEDGMENTS}

We thank Tristan Youngs for help with the NIMROD measurements and helpful conversations regarding the analysis. We also thank Tom Headen for all the help with the X-ray measurements and X-ray data corrections. This work benefited from the use of the SasView application, originally developed under NSF Award DMR-0520547. SasView contains code developed with funding from the European Union's Horizon 2020 research and innovation programme under the SINE2020 project, Grant Agreement No. 654000. Furthermore, we gratefully acknowledge the Science and Technology Facilities Council (STFC) for access to neutron beam time at ISIS on the NIMROD instrument (RB No. 1520094).

\section{ADDITIONAL NOTE}

${ }^{a}$ Because there are no forces between the atoms, molecules cannot cluster properly, but some molecules can still fulfill the clustering criteria due to their short intermolecular distances.

\section{REFERENCES}

(1) Olsson, C.; Jansson, H.; Youngs, T.; Swenson, J. Structure of aqueous trehalose solution by neutron diffraction and structural modeling. J. Phys. Chem. B 2016, 120, 12669-12678.

(2) Crowe, J. H.; Carpenter, J. F.; Crowe, L. M. The role of vitrification in anhydrobiosis. Annu. Rev. Physiol. 1998, 60, 73-103.

(3) Jain, N. K.; Roy, I. Effect of trehalose on protein structure. Protein Sci. 2008, 18, 24-36.

(4) Cordone, L.; Cottone, G.; Cupane, A.; Emanuele, A.; Giuffrida, S.; Levantino, M. Proteins in saccharides matrices and the trehalose peculiarity: biochemical and biophysical properties. Curr. Org. Chem. 2015, 19, 1684-1706.

(5) Bellavia, G.; Cottone, G.; Giuffrida, S.; Cupane, A.; Cordone, L. Thermal denaturation of myoglobin in water-disaccharide matrixes: relation with the glass transition of the system. J. Phys. Chem. B 2009, 113, 11543-11549.

(6) Olsson, C.; Jansson, H.; Swenson, J. The role of trehalose for the stabilization of proteins. J. Phys. Chem. B 2016, 120, 4723-4731.

(7) Crowe, J. H.; Crowe, L. M.; Jackson, S. A. Preservation of structural and functional activity in lyophilized sarcoplasmic reticulum. Arch. Biochem. Biophys. 1983, 220, 477-484.

(8) Crowe, J. H.; Crowe, L. M.; Carpenter, J. F.; Aurell Wistrom, C. Stabilization of dry phospholipid bilayers and proteins by sugars. Biochem. J. 1987, 242, 1-10.

(9) Crowe, L. M.; Womersley, C.; Crowe, J. H.; Reid, D.; Appel, L.; Rudolph, A. Prevention of fusion and leakage in freeze-dried liposomes by carbohydrates. Biochim. Biophys. Acta, Biomembr. 1986, 861, 131-140.

(10) Affouard, F.; Bordat, P.; Descamps, M.; Lerbret, A.; Magazù, S.; Migliardo, F.; Ramirez-Cuesta, A. J.; Telling, M. F. T. A combined neutron scattering and simulation study on bioprotectant systems. Chem. Phys. 2005, 317, 258-266.

(11) Magazu, S.; Migliardo, F.; Telling, M. T. F. Structural and dynamical properties of water in sugar mixtures. Food Chem. 2008, 106, 1460-1466.

(12) Lee, S. L.; Debenedetti, P. G.; Errington, J. R. A computational study of hydration, solution structure, and dynamics in dilute carbohydrate solutions. J. Chem. Phys. 2005, 122, 204511.

(13) Engelsen, S. B.; Monteiro, C.; Hervé de Penhoat, C.; Pérez, S. The diluted aqueous solvation of carbohydrates as inferred from molecular dynamics simulations and $\mathrm{nmr}$ spectroscopy. Biophys. Chem. 2001, 93, 103-127.

(14) Lerbret, A.; Bordat, P.; Affouard, F.; Descamps, M.; Migliardo, F. How homogeneous are the trehalose, maltose, and sucrose water solutions? an insight from molecular dynamics simulations. J. Phys. Chem. B 2005, 109, 11046-11057.

(15) Cottone, G. A comparative study of carboxy myoglobin in saccharide-water systems by molecular dynamics simulation. J. Phys. Chem. B 2007, 111, 3563-3569.

(16) Furuki, T. Effect of molecular structure on thermodynamic properties of carbohydrates. a calorimetric study of aqueous di- and oligosaccharides at subzero temperatures. Carbohydr. Res. 2002, 337, 441-450.

(17) Lammert, A. M.; Schmidt, S. J.; Day, G. A. Water activity and solubility of trehalose. Food Chem. 1998, 61, 139-144.

(18) Green, J. L.; Angell, C. A. Phase relations and vitrification in saccharide-water solutions and the trehalose anomaly. J. Phys. Chem. 1989, 93, 2880-2882.

(19) Crowe, L. M.; Reid, D. S.; Crowe, J. H. Is trehalose special for preserving dry biomaterials? Biophys. J. 1996, 71, 2087-2093.

(20) Malferrari, M.; Nalepa, A.; Venturoli, G.; Francia, F.; Lubitz, W.; Mobius, K.; Savitsky, A. Structural and dynamical characteristics of trehalose and sucrose matrices at different hydration levels as probed by ftir and high-field epr. Phys. Chem. Chem. Phys. 2014, 16, 9831-9848.

(21) Magazù, S.; Migliardo, F.; Telling, M. T. F. Study of the dynamical properties of water in disaccharide solutions. Eur. Biophys. J. 2007, 36, 163-171.

(22) Kilburn, D.; Townrow, S.; Meunier, V.; Richardson, R.; Alam, A.; Ubbink, J. Organization and mobility of water in amorphous and crystalline trehalose. Nat. Mater. 2006, 5, 632-635.

(23) Librizzi, F.; Vitrano, E.; Cordone, L. Dehydration and crystallization of trehalose and sucrose glasses containing carbonmonoxy-myoglobin. Biophys. J. 1999, 76, 2727-2734.

(24) Sussich, F.; Skopec, C.; Brady, J.; Cesàro, A. Reversible dehydration of trehalose and anhydrobiosis: from solution state to an exotic crystal? Carbohydr. Res. 2001, 334, 165-176.

(25) Magazù, S.; Migliardo, F.; Telling, M. T. F. Structural and dynamical properties of water in sugar mixtures. Food Chem. 2008, 106, 1460-1466.

(26) Branca, C.; Magazù, S.; Maisano, G.; Migliardo, F.; Migliardo, P.; Romeo, G. $\alpha, \alpha$-trehalose/water solutions. 5. hydration and viscosity in dilute and semidilute disaccharide solutions. J. Phys. Chem. B 2001, 105, 10140-10145.

(27) Magazu, S.; Villari, V.; Migliardo, P.; Maisano, G.; Telling, M. T. F.; Middendorf, H. D. Quasielastic neutron scattering study on disaccharide aqueous solutions. Phys. B 2001, 301, 130-133.

(28) Branca, C.; Maccarrone, S.; Magazù, S.; Maisano, G.; Bennington, S. M.; Taylor, J. Tetrahedral order in homologous disaccharide-water mixtures. J. Chem. Phys. 2005, 122, 174513.

(29) Branca, C.; Magazu, V.; Maisano, G.; Migliardo, F.; Soper, A. K. Study on destructuring effect of trehalose on water by neutron diffraction. Appl. Phys. A: Mater. Sci. Process. 2002, 74, s450-s451.

(30) Branca, C.; Magazu, S.; Migliardo, F.; Migliardo, P. Destructuring effect of trehalose on the tetrahedral network of water: a raman and neutron diffraction comparison. Phys. A 2002, 304, 314-318. 
(31) Bordat, P.; Lerbret, A.; Demaret, J. P.; Affouard, F.; Descamps, M. Comparative study of trehalose, sucrose and maltose in water solutions by molecular modelling. EPL (Europhysics Lett. 2004, 65, 41. (32) Shiraga, K.; Adachi, A.; Ogawa, Y. Characterization of the hydrogen-bond network of water around sucrose and trehalose: h-o-h bending analysis. Chem. Phys. Lett. 2017, 678, 59-64.

(33) Pagnotta, S. E.; McLain, S. E.; Soper, A. K.; Bruni, F.; Ricci, M. A. Water and trehalose: how much do they interact with each other? $J$. Phys. Chem. B 2010, 114, 4904-4908.

(34) Soper, A. K.; Ricci, M. A.; Bruni, F.; Rhys, N. H.; McLain, S. E. Trehalose in water revisited. J. Phys. Chem. B 2018, 122, 7365-7374.

(35) Bowron, D. T.; Soper, A. K.; Jones, K.; Ansell, S.; Birch, S.; Norris, J.; Perrott, L.; Riedel, D.; Rhodes, N. J.; Wakefield, S. R.; Botti, A.; Ricci, M.-A.; Grazzi, F.; Zoppi, M. NIMROD: the near and intermediate range order diffractometer of the isis second target station. Rev. Sci. Instrum. 2010, 81, 033905.

(36) Disordered materials software homepage, 2016.

(37) Soper, A. K. GudrunN and GudrunX - Programs for Correcting Raw Neutron and $x$-Ray Total Scattering Data to Differential Cross Section, 2012.

(38) Soper, A. K. Empirical potential monte carlo simulation of fluid structure. Chem. Phys. 1996, 202, 295-306.

(39) Damm, W.; Frontera, A.; Tirado-Rives, J.; Jorgensen, W. L. OPLS all-atom force field for carbohydrates. J. Comput. Chem. 1997, 18, 1955-1970.

(40) Berendsen, H. J. C.; Grigera, J. R.; Straatsma, T. P. The missing term in effective pair potentials. J. Phys. Chem. 1987, 91, 6269-6271.

(41) Pagnotta, S. E.; Ricci, M. A.; Bruni, F.; McLain, S.; Magazu, S. Water structure around trehalose. Chem. Phys. 2008, 345, 159-163.

(42) Towey, J. J.; Soper, A. K.; Dougan, L. Molecular insight into the hydrogen bonding and micro-segregation of a cryoprotectant molecule. J. Phys. Chem. B 2012, 116, 13898-13904.

(43) O’Dell, W. B.; Baker, D. C.; McLain, S. E. Structural evidence for inter-residue hydrogen bonding observed for cellobiose in aqueous solution. PLoS One 2012, 7, No. e45311.

(44) Conrad, P. B.; de Pablo, J. J. Computer simulation of the cryoprotectant disaccharide $\alpha, \alpha$-trehalose in aqueous solution. J. Phys. Chem. A 1999, 103, 4049-4055.

(45) Humphrey, W.; Dalke, A.; Schulten, K. VMD: visual molecular dynamics. J. Mol. Graphics 1996, 14, 33-38.

(46) SasView Software Website http://www.sasview.org/ (accessed March 1, 2018).

(47) Soper, A. K. The radial distribution functions of water as derived from radiation total scattering experiments: is there anything we can say for sure? ISRN Phys. Chem. 2013, 2013, 1.

(48) Mason, P. E.; Neilson, G. W.; Enderby, J. E.; Saboungi, M.-L.; Cuello, G.; Brady, J. W. Neutron diffraction and simulation studies of the exocyclic hydroxymethyl conformation of glucose. J. Chem. Phys. 2006, 125, 224505.

(49) Neilson, G. W.; Enderby, J. E. Aqueous solutions and neutron scattering. J. Phys. Chem. 1996, 100, 1317-1322.

(50) Soper, A. K. The radial distribution functions of water and ice from 220 to $673 \mathrm{k}$ and at pressures up to $400 \mathrm{mpa}$. Chem. Phys. 2000, 258, 121-137.

(51) Choi, Y.; Cho, K. W.; Jeong, K.; Jung, S. Molecular dynamics simulations of trehalose as a 'dynamic reducer'for solvent water molecules in the hydration shell. Carbohydr. Res. 2006, 341, 10201028.

(52) Rhys, N. H.; Bruni, F.; Imberti, S.; McLain, S. E.; Ricci, M. A. Glucose and mannose: a link between hydration and sweetness. J. Phys. Chem. B 2017, 121, 7771-7776.

(53) Towey, J. J.; Soper, A. K.; Dougan, L. What happens to the structure of water in cryoprotectant solutions? Faraday Discuss. 2014, 167, 159-176.

(54) Dhabal, D.; Singh, M.; Wikfeldt, K. T.; Chakravarty, C. Triplet correlation functions in liquid water. J. Chem. Phys. 2014, 141, 174504.
(55) Mathlouthi, M. X-ray diffraction study of the molecular association in aqueous solutions of d-fructose, d-glucose, and sucrose. Carbohydr. Res. 1981, 91, 113-123. 